\title{
Efficient regio- and stereoselective access to novel fluorinated $\beta$-aminocyclohexanecarboxylates
}

\author{
Loránd Kiss ${ }^{1}$, Melinda Nonn ${ }^{2}$, Reijo Sillanpää ${ }^{3}$, Santos Fustero ${ }^{4}$ \\ and Ferenc Fülöp*1,2
}

\section{Full Research Paper}

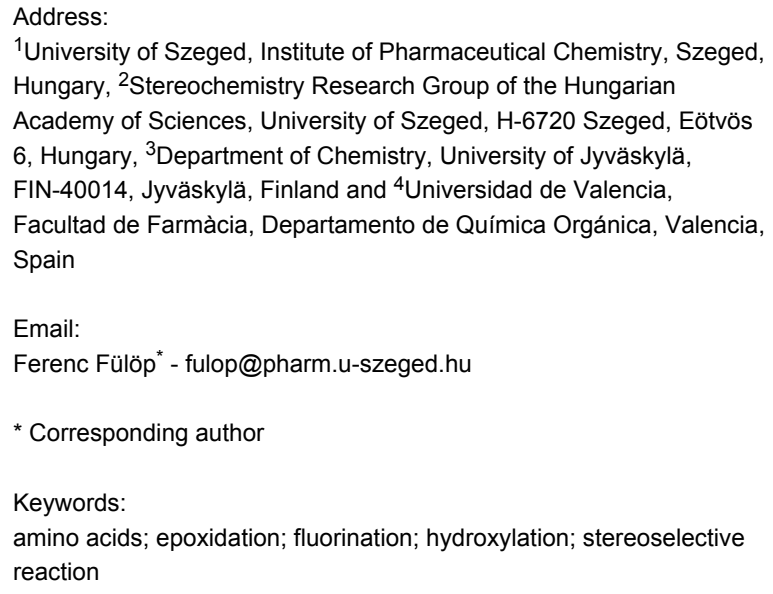

${ }^{1}$ University of Szeged, Institute of Pharmaceutical Chemistry, Szeged, Hungary, ${ }^{2}$ Stereochemistry Research Group of the Hungarian Academy of Sciences, University of Szeged, H-6720 Szeged, Eötvös 6, Hungary, ${ }^{3}$ Department of Chemistry, University of Jyväskylä, FIN-40014, Jyväskylä, Finland and ${ }^{4}$ Universidad de Valencia, Facultad de Farmàcia, Departamento de Química Orgánica, Valencia, Spain

Email:

Ferenc Fülöp* - fulop@pharm.u-szeged.hu

* Corresponding author

Keywords:

amino acids; epoxidation; fluorination; hydroxylation; stereoselective reaction

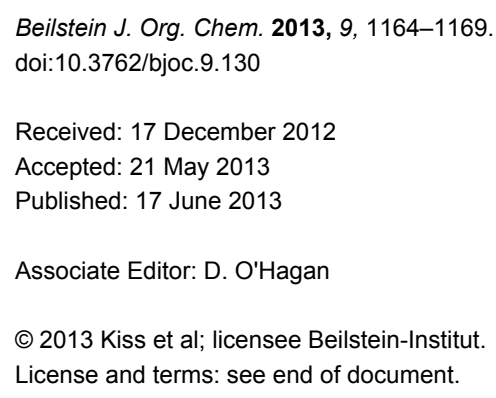

.


chemistry and in peptide research [36-60], only a relatively small number of fluorinated derivatives of this class of compounds have been synthesized so far [61-70].

\section{Results and Discussion}

We recently developed a synthetic method for the regio- and stereoselective introduction of a fluorine atom onto the skeleton of a $\beta$-aminocyclohexanecarboxylic acid. The synthesis starts from the Boc-protected 2-aminocyclohex-4-enecarboxylic acid or 2-aminocyclohex-3-enecarboxylic acid and involves ring $\mathrm{C}-\mathrm{C}$ bond transformation by regio- and stereoselective hydroxylation via iodolactonization, followed by hydroxy-fluorine exchange. This protocol was applied to synthesize fluorinated $\beta$-aminocyclohexane scaffolds with the fluorine atom on either position 3 or 5 of the ring. Whereas the procedure is a convenient economical route to fluorinated cyclohexane or cyclohexene $\beta$-amino acids, it did not allow extension to the synthesis of similar derivatives with the fluorine atom on position 4.

During our work performed to fill this gap, we have developed a synthetic procedure for gaining access to fluorinated $\beta$-aminocyclohexanecarboxylic acids.

This synthesis starts from ethyl cis-2-aminocyclohex-4-enecarboxylate 1 [57] and follows two different strategies. One is based on regio- and stereoselective hydroxylation via iodooxazine formation, followed by fluorination, while the other includes stereoselective epoxidation and regioselective oxirane opening, followed by hydroxy-fluorine exchange. In the former protocol, amino ester 1 is treated with $\mathrm{KI} / \mathrm{I}_{2}$ in $\mathrm{H}_{2} \mathrm{O} / \mathrm{CH}_{2} \mathrm{Cl}_{2}$, which affords iodooxazinone derivative 2 stereo- and regioselectively (Scheme 1, Figure 1). Next, compound $\mathbf{2}$ is transformed to 3 by amide $N$-Boc protection with $\mathrm{Boc}_{2} \mathrm{O}$ and 4-dimethylaminopyridine (DMAP) in THF. Removal of the iodine from the cyclohexane skeleton in $\mathbf{3}$ is accomplished under reductive conditions. On treatment with $n$ - $\mathrm{Bu}_{3} \mathrm{SnH}$ in the presence of a catalytic amount of azobisisobutyronitrile (AIBN) in dichloromethane under reflux, 3 undergoes deiodination to give ester 4 in $70 \%$ yield. Oxazinone 4 is then subjected to heterocycle ring opening with $\mathrm{NaOEt}$ in $\mathrm{EtOH}$ at $0{ }^{\circ} \mathrm{C}$ to furnish all-cis hydroxylated amino ester $\mathbf{5}$ with the hydroxy group on position 4 of the skeleton (Scheme 1, Figure 2).

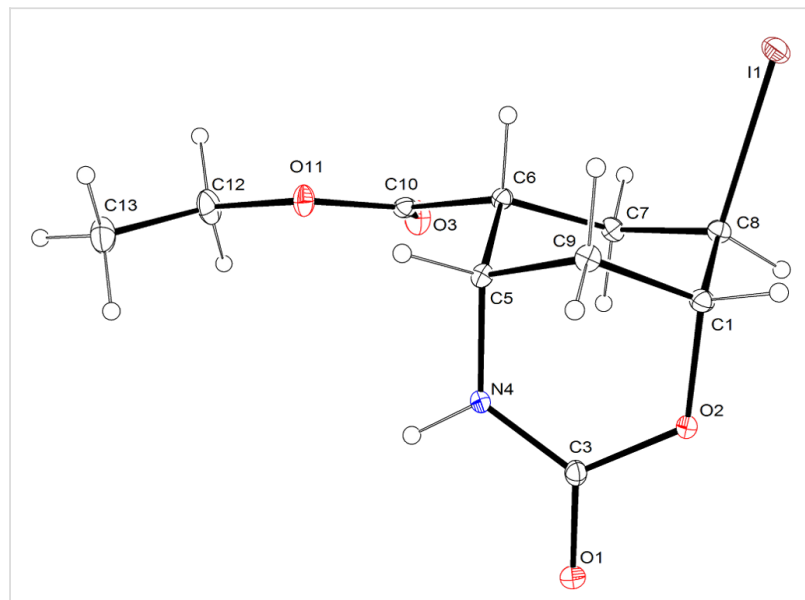

Figure 1: ORTEP diagram of iodooxazinone 2. Thermal ellipsoids have been drawn at the $20 \%$ probability level.

Hydroxylated amino ester 5 was also prepared via the alternative route involving stereoselective epoxidation. Cyclohexene $\beta$-amino ester 1 underwent $\mathrm{C}-\mathrm{C}$ double bond oxidation with 3-chloroperbenzoic acid (MCPBA) to afford epoxy amino ester 6 cis-diastereoselectively [57] (Scheme 1). Opening of the oxirane ring in 6 with $\mathrm{NaBH}_{4}$ in EtOH at $70{ }^{\circ} \mathrm{C}$ proceeded regioselectively, providing exclusively amino ester $\mathbf{5}$ with the<smiles>CCOC(=O)C1CC=CCC1NC(C)(C)C</smiles>

1

MCPBA, $\mathrm{CH}_{2} \mathrm{Cl}_{2}$

$0{ }^{\circ} \mathrm{C}, 6 \mathrm{~h}, 61 \%$<smiles>CCOC(=O)C1CC2OC2CC1NC(=O)OCc1ccccc1</smiles>

6

$$
\underset{\mathrm{H}_{2} \mathrm{O}, \mathrm{rt}, 18 \mathrm{~h}}{\stackrel{\mathrm{KI}, \mathrm{I}_{2}, \mathrm{CH}_{2} \mathrm{Cl}_{2}}{\longrightarrow}}
$$
$76 \%$<smiles>CCOC(=O)C1C[C@H](I)C2CC1NC(=O)O2</smiles>

2

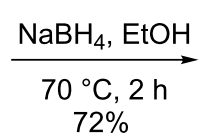<smiles>CCOC(=O)C1CCC(O)CC1NC(=O)OCc1ccccc1</smiles>

5

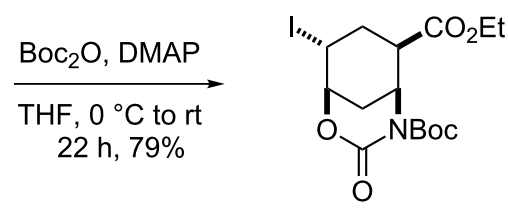

3

$n-\mathrm{Bu}_{3} \mathrm{SnH}, \mathrm{AlBN}$ $\mathrm{CH}_{2} \mathrm{Cl}_{2}, 40^{\circ} \mathrm{C}, 8 \mathrm{~h}, 70 \%$

$\mathrm{NaOEt}, \mathrm{EtOH}$

$0{ }^{\circ} \mathrm{C}, 1 \mathrm{~h}$ then $\mathrm{H}_{2} \mathrm{O}, 61 \%$

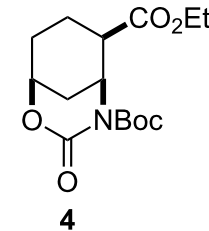




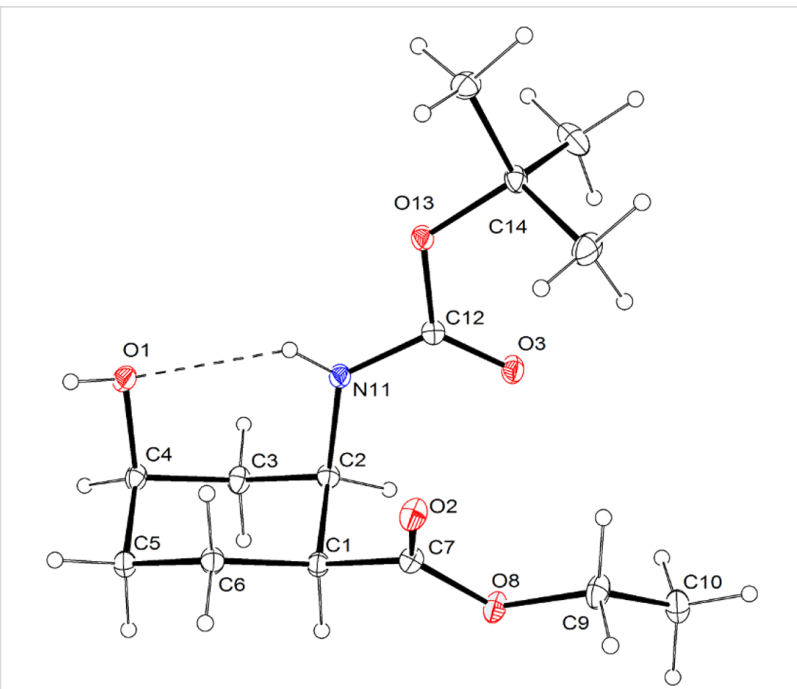

Figure 2: ORTEP diagram of hydroxylated amino ester $\mathbf{5}$. Thermal ellipsoids have been drawn at the $20 \%$ probability level.

hydroxy function on position 4 (for analogous transformations, see reference [60]).

Hydroxylated amino ester $\mathbf{5}$ was next further used as a key compound for the synthesis of fluorinated target materials. A fluorine atom was introduced by hydroxy-fluorine exchange with bis(dimethoxyethylaminosulfur trifluoride) (Deoxo-Fluor ${ }^{\circledR}$ ) reagent. The reaction was carried out under different experimental conditions, with variation of the temperature $\left(-40{ }^{\circ} \mathrm{C}\right.$, $0{ }^{\circ} \mathrm{C}$ or $20^{\circ} \mathrm{C}$ ) and the solvent (toluene, $\mathrm{CH}_{2} \mathrm{Cl}_{2}$ or THF). Finally, it was found that hydroxylated amino ester 5 underwent inversion on reaction with a $50 \%$ Deoxo-Fluor toluene solution in $\mathrm{CH}_{2} \mathrm{Cl}_{2}$ at $0{ }^{\circ} \mathrm{C}[68,69]$ to give monofluorinated cyclohexane amino ester 7 in $32 \%$ yield (Scheme 2). This rather modest yield is attributed to the relatively large amount of elim- ination materials ( $40 \%$ overall). In continuation, the geminal difluorinated $\beta$-aminocyclohexanecarboxylic acid derivative with the fluorine atoms on position 4 was efficiently synthesized. Oxidation of the hydroxy group of amino ester $\mathbf{5}$ with pyridinium chlorochromate ( $\mathrm{PCC}$ ) in $\mathrm{CH}_{2} \mathrm{Cl}_{2}$ yielded the corresponding oxo-group-containing amino ester $\mathbf{8}$, which was then converted with Deoxo-Fluor in $\mathrm{CH}_{2} \mathrm{Cl}_{2}$ at $0{ }^{\circ} \mathrm{C}$ to the corresponding geminal difluoro amino ester 9 in good yield (Scheme 2).

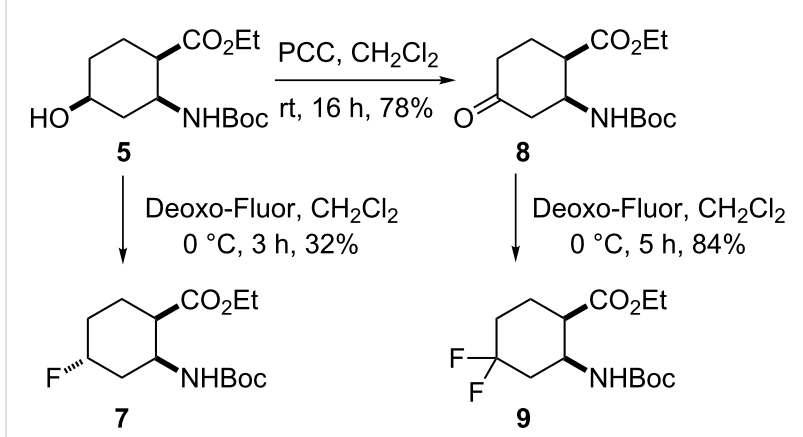

Scheme 2: Syntheses of fluorinated amino esters 7 and 9.

The synthetic route presented above could be extended to the preparation of other 4-fluorinated cyclohexane amino acid derivatives, stereoisomers of 7 or 9. Ethyl trans-2-aminocyclohex-4-enecarboxylate 10 [57] was analogously transformed to its cis counterpart through regio- and stereoselective iodooxazine formation with $\mathrm{KI} / \mathrm{I}_{2}$ to give compound $\mathbf{1 1}$ (Scheme 3). $\mathrm{N}$-Protection of $\mathbf{1 1}$, followed by reductive deiodination, proceeded via 12 (Figure 3) to afford ester 13. Opening of the heterocyclic ring with $\mathrm{NaOEt}$ in $\mathrm{EtOH}$ at $0{ }^{\circ} \mathrm{C}$ furnished 4-hydroxylated amino ester 14, a stereoisomer of 5 (Scheme 3, Figure 4).<smiles>CCOC(=O)[C@H]1CC=CCC1NC(C)(C)C</smiles>

10<smiles>CCOC(=O)[C@H]1CC2OC2CC1N[R6](=O)OCc1ccccc1</smiles>

15

$$
\frac{\mathrm{KI}, \mathrm{I}_{2}, \mathrm{CH}_{2} \mathrm{Cl}_{2}}{\underset{\substack{\mathrm{H}_{2} \mathrm{O}, \mathrm{rt}, 18 \mathrm{~h} \\ 73 \%}}{\longrightarrow}}
$$

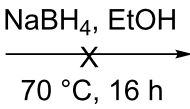

$70^{\circ} \mathrm{C}, 16 \mathrm{~h}$<smiles>CCO[C@H]1CCC(O)CC1N[R6](=O)OCc1ccccc1</smiles>

14<smiles>CCOC(=O)[C@@H]1C[C@H](I)[C@H]2C[C@H]1NC(=O)O2</smiles>

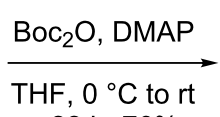
22 h, $76 \%$<smiles>CCOC(=O)[C@@H]1C[C@H](I)C2CC1[N+](C(=O)OCc1ccccc1)C(=O)O2</smiles>

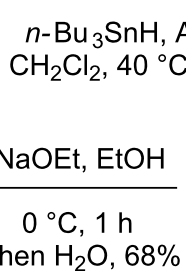

12

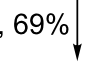<smiles>CCO[C@H]1CCC2CC1[N+](C(C)(C)C)C(=O)O2</smiles>

13 


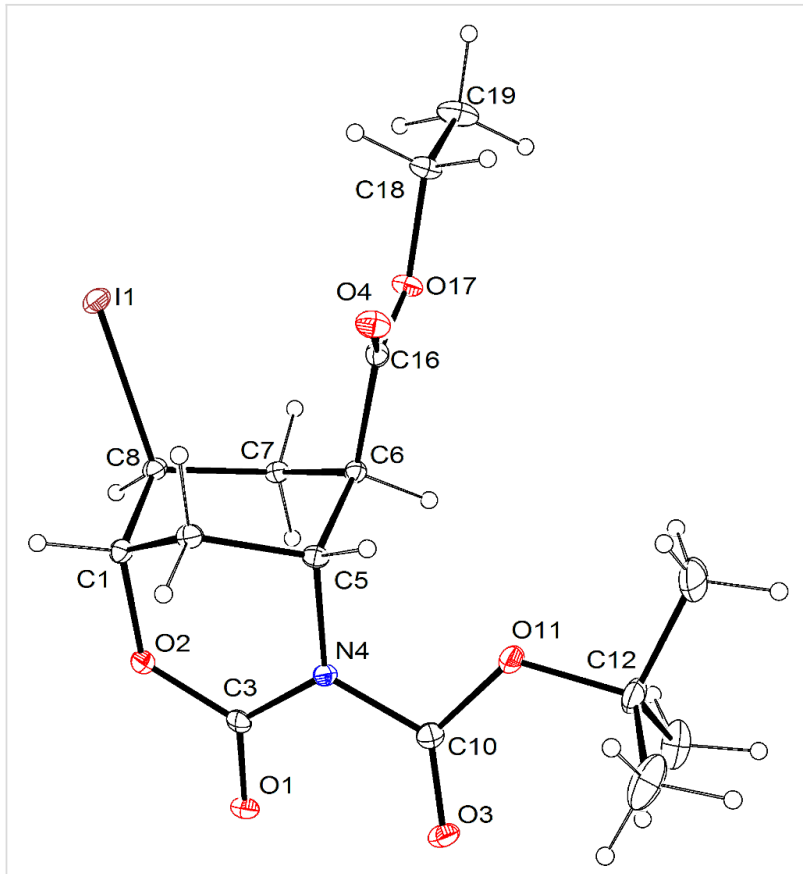

Figure 3: ORTEP diagram of iodooxazinone derivative 12. Thermal ellipsoids have been drawn at the $20 \%$ probability level.

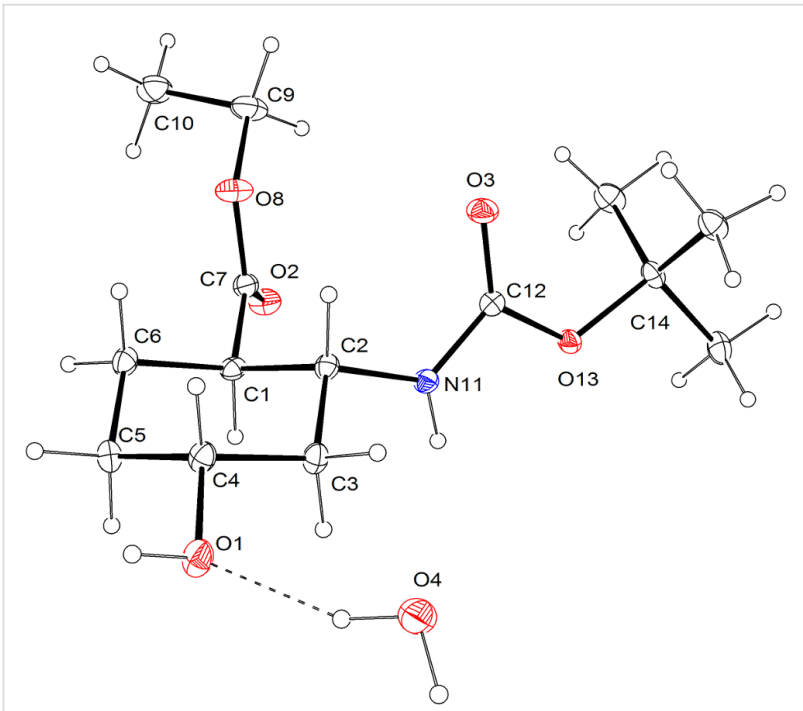

Figure 4: ORTEP diagram of hydroxylated amino ester 14. The water molecule oxygen atom $\mathrm{O} 4$ is situated on the twofold axis with a population parameter of 0.6. Thermal ellipsoids have been drawn at the $20 \%$ probability level.

It is noteworthy that in this latter case hydroxylated amino ester $\mathbf{1 4}$ could not be prepared by the alternative diastereoselective epoxidation and regioselective oxirane opening strategy: according to our previous results, the opening of epoxide $\mathbf{1 5}$ derived from 10 proceeded via a trans-diaxial chair conformation with the nucleophile attack on $\mathrm{C} 4$, thereby providing the 5-hydroxylated derivative $[57,60]$.
Next, hydroxylated cyclohexane amino ester 14 was converted with Deoxo-Fluor in $\mathrm{CH}_{2} \mathrm{Cl}_{2}$ at $0{ }^{\circ} \mathrm{C}$ to 4 -fluorinated ethyl $\beta$-aminocyclohexanecarboxylate 16, a stereoisomer of $\mathbf{7}$. Unfortunately, the formation of a substantial amount of a mixture of elimination material could again not be avoided. When subjected to oxidation with PCC, amino ester $\mathbf{1 4}$ gave the corresponding oxo ester 17, treatment of which with DeoxoFluor in $\mathrm{CH}_{2} \mathrm{Cl}_{2}$ at $0{ }^{\circ} \mathrm{C}$ provided geminal 4,4-difluorinated cyclohexane amino ester $\mathbf{1 8}$, a stereoisomer of $\mathbf{9}$ (Scheme 4).

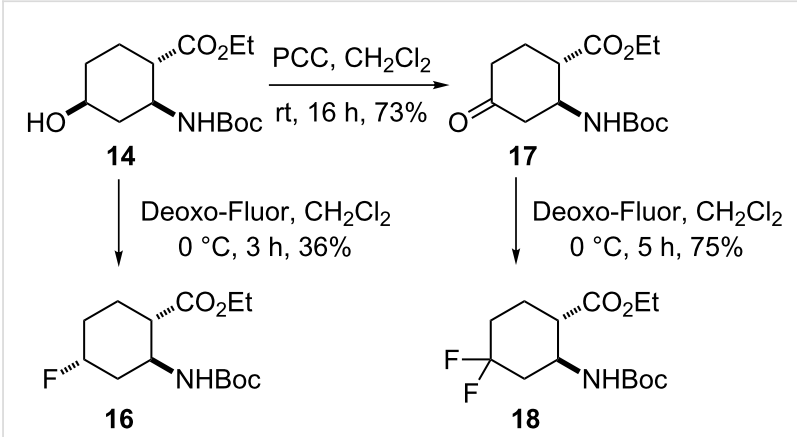

Scheme 4: Syntheses of fluorinated amino esters 16 and 18.

\section{Conclusion}

In conclusion, a simple and convenient procedure has been developed for the introduction of one or two fluorine atoms onto the skeleton of either cis- or trans- $\beta$-aminocyclohexanecarboxylates. The synthetic concept involves regio- and stereoselective hydroxylation via iodooxazine formation, followed by hydroxy-fluorine or oxo-fluorine exchange.

\section{Supporting Information}

\section{Supporting Information File 1}

Experimental procedures and characterization of compounds.

[http://www.beilstein-journals.org/bjoc/content/ supplementary/1860-5397-9-130-S1.pdf]

\section{Acknowledgements}

We are grateful to the Hungarian Research Foundation (OTKA No. NK81371 and K100530) and TAMOP-4.2.2.A-II/1/KONV2012-0052 for financial support.

\section{References}

1. Gouverneur, V.; Müller, K., Eds. Fluorine in Pharmaceutical and Medicinal Chemistry: From Biophysical Aspects to Clinical Applications; Imperial College Press: London, 2012.

2. Hagmann, W. K. J. Med. Chem. 2008, 51, 4359. doi:10.1021/jm800219f 
3. Isanbor, C.; O'Hagan, D. J. Fluorine Chem. 2006, 127, 303. doi:10.1016/j.jfluchem.2006.01.011

4. Fustero, S.; Sanz-Cervera, J. F.; Aceña, J. L.; Sánchez-Roselló, M. Synlett 2009, 525. doi:10.1055/s-0028-1087806

5. Kirk, K. L. J. Fluorine Chem. 2006, 127, 1013. doi:10.1016/j.jluchem.2006.06.007

6. O'Hagan, D. J. Fluorine Chem. 2010, 131, 1071. doi:10.1016/j.jfluchem.2010.03.003

7. Bégué, J.-P.; Bonnet-Delpon, D. J. Fluorine Chem. 2006, 127, 992. doi:10.1016/j.jfluchem.2006.05.006

8. Qiu, X.-L.; Xu, X.-H.; Qing, F.-L. Tetrahedron 2010, 66, 789. doi:10.1016/j.tet.2009.11.001

9. Purser, S.; Moore, P. R.; Swallow, S.; Gouverneur, V. Chem. Soc. Rev. 2008, 37, 320. doi:10.1039/b610213c

10. Bresciani, S.; Slawin, A. M. Z.; O'Hagan, D. J. Fluorine Chem. 2009, 130, 537. doi:10.1016/j.jfluchem.2009.03.003

11. Aceña, J. L.; Sorochinsky, A. E.; Soloshonok, V. A. Synthesis 2012, 44, 1591. doi:10.1055/s-0031-1289756

12. Lemonnier, G.; Lion, C.; Quirion, J.-C.; Pin, J.-P.; Goudet, C.; Jubault, P. Bioorg. Med. Chem. 2012, 20, 4716. doi:10.1016/j.bmc.2012.06.006

13. Salwiczek, M.; Nyakatura, E. K.; Gerling, U. I. M.; Ye, S.; Koksch, B. Chem. Soc. Rev. 2012, 41, 2135. doi:10.1039/c1cs15241f

14. Mykhailiuk, P. K.; Shishkina, S. V.; Shishkin, O. V.; Zaporozhets, O. A.; Komarov, I. V. Tetrahedron 2011, 67, 3091. doi:10.1016/j.tet.2011.02.082

15. Chia, P. W.; Livesey, M. R.; Slawin, A. M. Z.; van Mourik, T.; Wyllie, D. J. A.; O’Hagan, D. Chem.-Eur. J. 2012, 18, 8813. doi:10.1002/chem.201200071

16. Shibata, N.; Nishimine, T.; Shibata, N.; Tokunaga, E.; Kawada, K.; Kagawa, T.; Sorochinsky, A. E.; Soloshonok, V. A. Chem. Commun. 2012, 48, 4124. doi:10.1039/c2cc30627a

17. Smits, R.; Cadicamo, C. D.; Burger, K.; Koksch, B. Chem. Soc. Rev. 2008, 37, 1727. doi:10.1039/b800310f

18. Sorochinsky, A. E.; Soloshonok, V. A. J. Fluorine Chem. 2010, 131 127. doi:10.1016/j.jfluchem.2009.09.015

19. Pan, Y.; Zhao, Y.; Ma, T.; Yang, Y.; Liu, H.; Jiang, Z.; Tan, C.-H. Chem.-Eur. J. 2010, 16, 779. doi:10.1002/chem.200902830

20. Acena, J. L.; Simon-Fuentes, A.; Fustero, S. Curr. Org. Chem. 2010, 14, 928. doi:10.2174/138527210791111777

21. Tarui, A.; Sato, K.; Omote, M.; Kumadaki, I.; Ando, A. Adv. Synth. Catal. 2010, 352, 2733. doi:10.1002/adsc.201000506

22. Hook, D. F.; Gessier, F.; Noti, C.; Kast, P.; Seebach, D. ChemBioChem 2004, 5, 691. doi:10.1002/cbic.200300827

23. Jäckel, C.; Koksch, B. Eur. J. Org. Chem. 2005, 4483. doi:10.1002/ejoc.200500205

24. Yoder, N. C.; Kumar, K. Chem. Soc. Rev. 2002, 31, 335. doi:10.1039/b201097f

25. Mathad, R. I.; Jaun, B.; Flögel, O.; Gardiner, J.; Löweneck, M.; Codée, J. D. C.; Seeberger, P. H.; Seebach, D.; Edmonds, M. K.; Graichen, F. H. M.; Abell, A. D. Helv. Chim. Acta 2007, 90, 2251. doi:10.1002/hlca.200790235

26. Capone, S.; Kieltsch, I.; Flögel, O.; Lelais, G.; Togni, A.; Seebach, D. Helv. Chim. Acta 2008, 91, 2035. doi:10.1002/hlca.200890217

27. Fustero, S.; Sánchez-Roselló, M.; Rodrigo, V.; Sanz-Cervera, J. F.; Piera, J.; Simón-Fuentes, A.; del Pozo, C. Chem.-Eur. J. 2008, 14, 7019. doi:10.1002/chem.200702009

28. Fustero, S.; Sánchez-Roselló, M.; Rodrigo, V.; del Pozo, C.; Sanz-Cervera, J. F.; Simón, A. Org. Lett. 2006, 8, 4129. doi:10.1021/ol061733c
29. Mykhailiuk, P. K.; Radchenko, D. S.; Komarov, I. V. J. Fluorine Chem. 2010, 131, 221. doi:10.1016/j.jluchem.2009.07.017

30. Pan, Y.; Calvert, K.; Silverman, R. B. Bioorg. Med. Chem. 2004, 12, 5719. doi:10.1016/j.bmc.2004.07.065

31. Yasuhara, A.; Sakagami, K.; Yoshikawa, R.; Chaki, S.; Nakamura, M.; Nakazato, A. Bioorg. Med. Chem. 2006, 14, 3405. doi:10.1016/j.bmc.2005.12.061

32. Wang, Z.; Silverman, R. B. Bioorg. Med. Chem. 2006, 14, 2242. doi:10.1016/j.bmc.2005.11.010

33. Lu, H.; Silverman, R. B. J. Med. Chem. 2006, 49, 7404. doi:10.1021/jm0608715

34. Seebach, D.; Gardiner, J. Acc. Chem. Res. 2008, 41, 1366. doi:10.1021/ar700263g

35. Seebach, D. Angew. Chem., Int. Ed. 2011, 50, 96. doi:10.1002/anie.201003823

36. Fülöp, F. Chem. Rev. 2001, 101, 2181. doi:10.1021/cr000456z

37. Kiss, L.; Forró, E.; Fülöp, F. Synthesis of Carbocyclic $\beta$-Amino Acids. In Amino Acids, Peptides and Proteins in Organic Chemistry; Hughes, A. B., Ed.; Wiley-VCH: Weinheim, Germany, 2009; Vol. 1, pp 367 ff. doi:10.1002/9783527631766.ch8

38. Hameršak, Z.; Roje, M.; Avdagić, A.; Šunjić, V. Tetrahedron: Asymmetry 2007, 18, 635. doi:10.1016/j.tetasy.2007.02.019

39. Rathore, N.; Gellman, S. H.; de Pablo, J. J. Biophys. J. 2006, 91, 3425. doi:10.1529/biophysj.106.084491

40. Fernandes, C.; Gauzy, C.; Yang, Y.; Roy, O.; Pereira, E.; Faure, S.; Aitken, D. J. Synthesis 2007, 2222. doi:10.1055/s-2007-983759

41. Mowery, B. P.; Lee, S. E.; Kissounko, D. A.; Epand, R. F.; Epand, R. M.; Weisblum, B.; Stahl, S. S.; Gellman, S. H. J. Am. Chem. Soc. 2007, 129, 15474. doi:10.1021/ja077288d

42. Gorrea, E.; Nolis, P.; Torres, E.; Da Silva, E.; Amabilino, D. B.; Branchadell, V.; Ortuño, R. M. Chem.-Eur. J. 2011, 17, 4588. doi:10.1002/chem.201002193

43. Rúa, F.; Boussert, S.; Parella, T.; Díez-Pérez, I.; Branchadell, V.; Giralt, E.; Ortuño, R. M. Org. Lett. 2007, 9, 3643. doi:10.1021/ol701521k

44. Porter, E. A.; Weisblum, B.; Gellman, S. H. J. Am. Chem. Soc. 2005, 127, 11516. doi:10.1021/ja0519785

45. Roy, O.; Faure, S.; Aitken, D. J. Tetrahedron Lett. 2006, 47, 5981. doi:10.1016/j.tetlet.2006.06.027

46. D’Elia, V.; Zwicknagl, H.; Reiser, O. J. Org. Chem. 2008, 73, 3262. doi:10.1021/jo800168h

47. Hetényi, A.; Szakonyi, Z.; Mándity, I. M.; Szolnoki, É.; Tóth, G. K.; Martinek, T. A.; Fülöp, F. Chem. Commun. 2009, 177. doi:10.1039/b812114a

48. Fülöp, F.; Martinek, T. A.; Tóth, G. K. Chem. Soc. Rev. 2006, 35, 323. doi:10.1039/b501173f

49. Torres, E.; Acosta-Silva, C.; Rúa, F.; Álvarez-Larena, A.; Parella, T.; Branchadell, V.; Ortuño, R. M. Tetrahedron 2009, 65, 5669. doi:10.1016/j.tet.2009.05.039

50. Farnández, D.; Torres, E.; Avilés, F. X.; Ortuño, R. M.; Vendrell, J. Bioorg. Med. Chem. 2009, 17, 3824. doi:10.1016/j.bmc.2009.04.035

51. Fernandes, C.; Pereira, E.; Faure, S.; Aitken, D. J. J. Org. Chem. 2009, 74, 3217. doi:10.1021/jo900175p

52. Celis, S.; Gorrea, E.; Nolis, P.; Illa, O.; Ortuño, R. M. Org. Biomol. Chem. 2012, 10, 861. doi:10.1039/c1ob06575k

53. Szolnoki, É.; Hetényi, A.; Martinek, T. A.; Szakonyi, Z.; Fülöp, F. Org. Biomol. Chem. 2012, 10, 255. doi:10.1039/c1ob06627g

54. Martinek, T. A.; Fülöp, F. Chem. Soc. Rev. 2012, 41, 687. doi:10.1039/c1cs15097a 
55. Mansawat, W.; Vilaivan, C.; Balázs, Á.; Aitken, D. J.; Vilaivan, T.

Org. Lett. 2012, 14, 1440. doi:10.1021/ol300190u

56. Berlicki, Ł.; Pilsl, L.; Wéber, E.; Mándity, I. M.; Cabrele, C.;

Martinek, T. A.; Fülöp, F.; Reiser, O. Angew. Chem., Int. Ed. 2012, 51, 2208. doi:10.1002/anie.201107702

57. Kiss, L.; Forró, E.; Fülöp, F. Tetrahedron 2012, 68, 4438. doi:10.1016/j.tet.2011.12.065

58. Nonn, M.; Kiss, L.; Sillanpää, R.; Fülöp, F. Beilstein J. Org. Chem. 2012, 8, 100. doi:10.3762/bjoc.8.10

59. Kiss, L.; Forró, E.; Sillanpää, R.; Fülöp, F. Tetrahedron 2010, 66, 3599. doi:10.1016/j.tet.2010.03.030

60. Kiss, L.; Forró, E.; Martinek, T. A.; Bernáth, G.; De Kimpe, N.; Fülöp, F. Tetrahedron 2008, 64, 5036. doi:10.1016/j.tet.2008.03.068

61. Qiu, X.-L.; Qing, F.-L. Eur. J. Org. Chem. 2011, 3261.

doi:10.1002/ejoc.201100032

62. Mikami, K.; Fustero, S.; Sánchez-Roselló, M.; Aceña, J. L.; Soloshonok, V.; Sorochinsky, A. Synthesis 2011, 3045. doi:10.1055/s-0030-1260173

63. Fustero, S.; Sanz-Cervera, J. F.; Piera, J.; Sánchez-Roselló, M.; Chiva, G.; Simón-Fuentes, A. J. Fluorine Chem. 2004, 125, 621. doi:10.1016/j.jfluchem.2003.12.016

64. Fustero, S.; Sánchez-Roselló, M.; Aceña, J. L.; Fernández, B.; Asensio, A.; Sanz-Cervera, J. F.; del Pozo, C. J. Org. Chem. 2009, 74, 3414. doi:10.1021/jo900296d

65. Fustero, S.; Sánchez-Roselló, M.; Sanz-Cervera, J. F.; Aceña, J. L.; del Pozo, C.; Fernández, B.; Bartolomé, A.; Asensio, A. Org. Lett. 2006, 8, 4633. doi:10.1021/ol061892w

66. Mittendorf, J.; Kunisch, F.; Matzke, M.; Militzer, H.-C.; Schmidt, A.; Schönfeld, W. Bioorg. Med. Chem. Lett. 2003, 13, 433. doi:10.1016/S0960-894X(02)00958-7

67. Hamashima, Y.; Suzuki, T.; Takano, H.; Shimura, Y.; Tsuchiya, Y.; Moriya, K.; Goto, T.; Sodeoka, M. Tetrahedron 2006, 62, 7168. doi:10.1016/j.tet.2005.12.070

68. Kiss, L.; Forró, E.; Fustero, S.; Fülöp, F. Eur. J. Org. Chem. 2011, 4993. doi:10.1002/ejoc.201100583

69. Kiss, L.; Forró, E.; Fustero, S.; Fülöp, F. Org. Biomol. Chem. 2011, 9, 6528. doi:10.1039/c1ob05648d

70. Nonn, M.; Kiss, L.; Hänninen, M. M.; Sillanpää, R.; Fülöp, F. Chem. Biodiversity 2012, 9, 2571. doi:10.1002/cbdv.201200323

\section{License and Terms}

This is an Open Access article under the terms of the Creative Commons Attribution License (http://creativecommons.org/licenses/by/2.0), which permits unrestricted use, distribution, and reproduction in any medium, provided the original work is properly cited.

The license is subject to the Beilstein Journal of Organic Chemistry terms and conditions:

(http://www.beilstein-journals.org/bjoc)

The definitive version of this article is the electronic one which can be found at: doi:10.3762/bjoc. 9.130 